Suárez Lantarón, B., Castillo Reche, I. \& López Medialdea, A. (2021). Tutoría académica universitaria apoyada mediante WhatsApp: conocer sus ventajas y salvar sus desventajas. Revista Electrónica Interuniversitaria de Formación del Profesorado, 24(1), 189-203.

DOI: https://doi.org/10.6018/reifop.394631

\title{
Tutoría académica universitaria apoyada mediante WhatsApp: conocer sus ventajas y salvar sus desventajas
}

\author{
Belén Suárez Lantarón', Irina S. Castillo Reche ${ }^{2}$ y Ana López Medialdea ${ }^{1}$ \\ ${ }^{1}$ Universidad de Extremadura, ${ }^{2}$ Universidad de Murcia
}

\section{Resumen}

El presente artículo describe un proyecto de investigación e innovación docente que tiene como objetivo valorar el efecto del uso de la aplicación WhatsApp para el seguimiento y tutoría académica de los estudiantes universitarios de la Facultad de Educación de Murcia y de Extremadura. Los datos fueron recogidos a través de dos cuestionarios cumplimentados por los estudiantes antes y después del desarrollo de las asignaturas y aplicación del proyecto, un grupo de discusión con los docentes y el análisis del contenido de los mensajes enviados a través de la aplicación. Se analizaron los resultados obtenidos teniendo en cuenta la utilización y el uso de la aplicación, la satisfacción de los participantes y las ventajas e inconvenientes descritos. Los resultados muestran que se realiza un uso adecuado de la aplicación, se utiliza más que la tutoría convencional, se potencian sus ventajas y se debilitan sus inconvenientes y tanto las docentes como los estudiantes muestran altos niveles de satisfacción. Por tanto, podemos concluir que estamos ante una herramienta con un alto potencial para el apoyo a la tutoría académica.

\section{Palabras clave}

Tutoría académica; WhatsApp; universidad; innovación docente.

\section{University academic tutoring supported by WhatsApp: know its advantages and save its disadvantages}

\section{Abstract}

This paper describes a teaching research and innovation project whose aim is to assess the effect of using the WhatsApp application for monitoring and academic tutoring of university students of the Faculty of Education of Murcia and Extremadura. The data were 
collected through two questionnaires filled out by the students before and after the development of the subjects and application of the project, a discussion group with the teachers and the analysis of the content of the messages sent through the application. The results obtained were analysed taking into account the use and use of the application, the satisfaction of the participants and the advantages and disadvantages described. The results show that proper use of the application is made, more than conventional tutoring, its advantages are enhanced and its disadvantages are weakened and both teachers and students show high levels of satisfaction. Therefore, we can conclude that we are facing a tool with a high potential for supporting academic tutoring.

\section{Key words}

Academic tutoring; WhatsApp; University; Educational innovation

\section{Introducción/Justificación}

La relevancia de la acción tutorial en el ámbito de la educación superior ha quedado sobradamente reflejada en el extenso número de documentos que al respecto pueden encontrarse.

La Universidad, reconociendo el derecho de los estudiantes a la orientación durante su periodo formativo, apuesta por un sistema integrado y coordinado de acciones que apoyen y orienten al alumnado durante dicho proceso (Echeverría y Martínez, 2015) entre las que se encuentra la tutoría, como modo de respuesta a las necesidades de los estudiantes, procurando, de este modo, una atención más personalizada, que asuma la diversidad y multiculturalidad de las aulas de educación superior (Álvarez y Álvarez, 2015).

En el contexto en el que se desarrolla este trabajo, entendemos la tutoría como un proceso de apoyo y ayuda al estudiante de carácter formativo y orientador; además, dicho proceso debe contribuir a la formación integral del estudiante (García, 2010).

Desde esta perspectiva, la tutoría pasa a ser un proceso inherente a la función docente (Alonso, Rodríguez y Cáceres, 2018), y un componente esencial, que acompaña al alumnado en su proceso de aprendizaje (Echeverría y Martínez, 2015) facilitando una transición exitosa y favorecedora de la permanencia del estudiante en la universidad (Álvarez y Álvarez, 2015) y que puede desarrollarse de forma individual o grupal (García, 2010).

Según el estudio realizado por Alonso et al. (2018, p. 65), los alumnos se encuentran de acuerdo (49,7\% señalan esta opción, siendo el porcentaje más elevado) respecto a la utilidad de la tutoría para esclarecer aspectos del ámbito académico y sobre la utilidad de la acción tutorial universitaria para suplir los contenidos docentes, es decir, aquellos relacionados con la planificación y organización de la asignatura (48,3\% de acuerdo y $8,8 \%$ totalmente de acuerdo).

Sin embargo, en la práctica no siempre es entendida así y parece que no acaba de responder a las esperanzas puestas tanto por parte de las universidades como de los estudiantes (Echeverría y Martínez, 2015). Este aspecto es corroborado por los resultados del estudio realizado por Medialdea (2014, p.412) sobre la tutoría universitaria, dónde se plantea si ésta se adapta a las necesidades del alumnado, confirmando determinadas carencias en la orientación recibida durante el primer curso universitario, como es la coincidencia con horarios de clase $(42,42 \%)$ y la baja implicación del profesorado en el desarrollo de las sesiones tutoriales. Otros estudios, como el de Da Re y Clerici (2017) 
destacan el poco uso que el alumnado hace de este tipo de apoyos, localizándose en momentos puntuales, como la revisión de exámenes o trabajos (Suárez, 2017).

Por otro lado, las nuevas tecnologías (TIC) constituyen una herramienta decisiva en todos los órdenes de nuestras vidas (personal, social y profesional). El alumnado que accede a nuestras aulas ha crecido en un entorno tecnológico, en el que internet juega un papel decisivo permitiéndoles, entre otras cosas, estar conectados.

En palabras de Sonlleva, Torrego y Martínez (2017, p. 258):

Los dispositivos móviles, las videoconsolas, la televisión o las redes, constituyen, desde el punto de vista educativo, algo más que unos recursos susceptibles de ser utilizados en la enseñanza. Son para nuestros educandos, experiencia vital desde antes de entrar al aula.

Quizá por esta razón, los docentes aprovechan la predisposición de los estudiantes hacia este tipo de recursos para incorporarlos en sus actividades académicas, como herramientas para "acceder a vastos recursos de conocimiento, a colaborar con otros compañeros, consultar a expertos, compartir conocimiento o resolver problemas complejos" (Martínez y Raposo, 2006, p. 167).

Asimismo, si nos centramos en la aplicación WhatsApp (WA), son numerosos los estudios que muestran los efectos positivos de su uso como herramienta de comunicación e intercambio de información en tiempo real en el marco de la educación superior, al extender la posibilidad de compartir el conocimiento más allá de las paredes del aula. (Barhoumi, 2015; Gende, 2016; Rodríguez, Valerio, Cárdenas y Herrara, 2016).

Estas cuestiones -la importancia de la tutoría, el uso de las nuevas tecnologías y los beneficios identificados en investigaciones anteriores sobre el uso de WhatsApp- nos llevan a implementar un proyecto cuyo objetivo es comprobar si WhatsApp puede ser una herramienta adecuada de apoyo a la tutoría, así como sus ventajas y desventajas. Ya que, como señalan López y Prendes (2017), si en el proceso de tutoría incluimos el uso de las TIC, lograremos un modelo de acción tutorial más flexible y más ajustado a las necesidades del alumnado.

Dicho objetivo general, se concreta a través de los siguientes interrogantes más específicos:

(i) ¿utilizan los estudiantes la aplicación WhatsApp en el ámbito educativo?

(ii) ¿Cómo utilizan dicha herramienta?

Una vez presentada la aplicación como herramienta de apoyo a la tutoría:

(iii) ¿Qué uso dan a la aplicación?

(iv) ¿Es satisfactoria la experiencia para los participantes?

(v) Ventajas y desventajas observadas

\section{Metodología}

Para alcanzar el objetivo antes mencionado, la metodología utilizada ha sido mixta, utilizando herramientas cualitativas y cuantitativas. El proyecto, con carácter longitudinal, ha pasado por tres etapas diferenciadas: (i) Fase inicial: prueba piloto, (ii) Fase de control y (iii) Fase final, desarrolladas a lo largo de tres cursos académicos en la Facultad de Educación de la Universidad de Murcia. 
Exponemos de forma más detallada la muestra participante y el procedimiento seguido en cada una de ellas:

\section{Fase inicial: prueba piloto}

Esta primera fase se inicia en el curso 2016/17 con el objetivo de conocer si la aplicación WA resultar ser una herramienta adecuada de apoyo para la tutoría académica.

Para lograr dicho objetivo, se implementa una experiencia en la que participan un total de 70 estudiantes del primer curso del Grado de Educación Primaria y un docente.

Al inicio de curso, se propuso la formación de un grupo de WhatsApp específico para la asignatura de Organización Escolar y Recursos Educativos. En dicho grupo se incluía al profesor de la materia y se utilizaría, además de las tutorías establecidas (presencial y virtual), como medio para consultar dudas que surgieran durante el proceso de enseñanzaaprendizaje.

Recordemos que las cuestiones a las que la experiencia pretende dar respuesta son, por un lado:

(i) ¿utilizan los estudiantes la aplicación WA en el ámbito educativo?

(ii) ¿Cómo utilizan dicha herramienta?

Una vez presentada la aplicación como herramienta de apoyo a la tutoría:

(iii) ¿Qué uso dan a la aplicación?

(iv) ¿Es satisfactoria la experiencia para los participantes?

(v) Ventajas y desventajas observadas

Para obtener la información y con el fin de conseguir una triangulación de esta, se utiliza una metodología tanto cuantitativa como cualitativa, utilizando para ello la técnica de encuesta a través de la elaboración de un cuestionario inicial y final para el alumnado (ver anexo I y II), fundamentado en la revisión de la literatura sobre el tema objeto de estudio; se utiliza también un grupo de discusión (docente-estudiantes) para profundizar en la información obtenida a través de los cuestionarios; y, además, para conocer el uso dado a la aplicación, el docente registra los mensajes/contenidos tratados a través del grupo de WA para su posterior análisis.

\section{Fase de control}

Esta segunda fase se realizó durante el curso 2017/18 con el objetivo de comprobar si realmente el uso de la aplicación WA como apoyo a la tutoría ayuda en la mejora de los resultados académicos de los estudiantes.

Para lograr esta meta se llevó a cabo una réplica de la experiencia anterior. En esta ocasión participaron dos grupos de estudiantes ( 72 y 70 estudiantes, respectivamente) del mismo nivel, grado y misma asignatura, y un docente que impartía la asignatura en ambos grupos.

El proyecto consistió en utilizar en uno de los grupos WhatsApp del mismo modo que en la experiencia previa y en el otro (grupo de control) únicamente los medios de tutoría ordinarios (tutoría virtual y presencial). Los datos se recogen a través de los mismos cuestionarios presentados al alumnado en la prueba piloto y la observación y experiencia relatada por el docente (registro de observación-diario de aula). 


\section{Fase final}

La tercera fase del proyecto tuvo lugar durante el curso 2018/19 bajo el formato de proyecto de innovación docente (aprobado en la convocatoria que la Universidad de Murcia formuló en ese periodo).

El objetivo planteado era comprobar la utilidad de la aplicación siendo utilizada por más docentes y estudiantes, así como la satisfacción de estos con el uso de la aplicación. Además, en esta tercera fase se busca salvar algunas de las desventajas observadas en las fases anteriores:

- Uso inadecuado por parte de los estudiantes

- Uso en horas no pertinentes para las consultas

- Recelo del docente en la utilización de su número personal.

Para ello se redacta un contrato de compromiso que el alumnado participante debe firmar (ver anexo III) en el que se detallan las normas de uso del grupo, así como el compromiso con la protección de datos (números personales de teléfono) e información (contenido de las conversaciones). Todo estudiante que quisiera ser incluido en el grupo debía presentar este contrato firmado al tutor.

En esta tercera fase, participaron dos docentes de la Universidad de Murcia y una de la Universidad de Extremadura y se incrementó el número de asignaturas en las que se utilizó WA como herramienta de apoyo a la tutoría. También el número de los estudiantes que participaron fue mayor.

La muestra de estudiantes que se incluyen en los grupos de WA y su distribución por asignaturas, se recogen en la tabla 2.

Tabla 2.

Estudiantes distribuidos por grupos/asignaturas

\begin{tabular}{|c|c|c|}
\hline NOMBRE DE LA ASIGNATURA & $\begin{array}{l}\mathrm{N}^{\circ} \text { de } \\
\text { alumnos } \\
\text { matriculados }\end{array}$ & $\begin{array}{l}\mathrm{N}^{\circ} \text { de } \\
\text { alumnos } \\
\text { incluidos en } \\
\text { WA }\end{array}$ \\
\hline Evaluación de servicios y profesionales de la Educación Social & 52 & 45 \\
\hline Procesos y condiciones para el desarrollo de proyectos & 64 & 52 \\
\hline Prácticas externas I (Grado de E. Social) & 4 & 4 \\
\hline Prácticas externas I (Grado de Pedagogía) & 5 & 5 \\
\hline Prácticas Escolares I (Grado de E. Primaria) & 8 & 8 \\
\hline Tutorización de TFM- Línea Orientación & 1 & 1 \\
\hline Practicum II- Atención Temprana & 17 & 17 \\
\hline Tota & 151 & 132 \\
\hline
\end{tabular}

Para conocer el uso dado por los estudiantes, así como su satisfacción se utilizaron los mismos cuestionarios anteriores. La satisfacción de los docentes participantes con el uso de esta aplicación es valorada utilizando el grupo de discusión. Este medio nos permite 
conocer el grado de satisfacción, las sensaciones percibidas, así como las ventajas y desventajas observadas por dichos docentes.

\section{Resultados y discusión}

Los resultados obtenidos se presentan en base a los interrogantes planteados en las diferentes fases del proyecto.

Previamente al desarrollo de la experiencia, buscamos cuál es el que dan a la aplicación los estudiantes universitarios:

\section{(i) ¿Utilizan los estudiantes la aplicación WA en el ámbito educativo?}

Los datos indican que el $100 \%$ de los estudiantes que han participado en el proyecto utiliza su teléfono para estar conectado, dedicando una media de 12 horas (siendo el máximo recogido de 16 y el mínimo de 4).

Utilizan WA como mensajería el 100\% de los participantes, además de Facebook (92\%) e Instagram (85\%).

Estos datos no hacen más que confirmar los obtenidos en otros informes o estudios previos sobre la aplicación WhatsApp que la sitúan como la más utilizada por parte de los jóvenes (Ditrendia, 2018; Rubio y Perlado, 2015; Sonlleva et al., 2017)

Para nuestros estudiantes WhatsApp ya no es sólo un medio de comunicación interpersonal entre ellos sino que "hablar a través de esta aplicación se ha convertido en una forma de comunicarse; un espacio donde se tienen experiencias" (Rubio y Perlado, 2015, p. 85).

En relación con el uso educativo que dan a la aplicación, el 98\% indica utilizar esta aplicación para intercambiar información sobre el curso (aspectos académicos) principalmente con los compañeros (100\%) y los amigos (95\%). Además, señalan tener prácticamente un grupo por cada una de las asignaturas en las que están matriculados, aunque en ninguno de ellos se incluye al docente.

Además, el 98.6\% reconoce que esta aplicación puede resultar útil en su proceso de enseñanza-aprendizaje. Entre las ventajas que señalan: compartir información de forma rápida y poder solucionar las dudas que surgen; entre las desventajas: que tener tantos grupos implica recibir muchos mensajes, algunos poco útiles, a los que se debe dedicar tiempo que podría emplearse para otra cosa.

Este último aspecto coincide con lo expuesto por Rubio y Perlado (2017). Los jóvenes que participaron en su estudio destacan, en relación al uso de WhatsApp, una "cierta compulsión a estar permanentemente comunicándose lo que motiva una cierta frivolidad conversacional" (p. 84).

\section{(ii) ¿Cómo utilizan dicha herramienta?}

Los estudiantes señalan que el uso educativo que suelen dar a esta aplicación a través de los grupos de las asignaturas es:

- En un $98,7 \%$ para consultar dudas sobre lo explicado en clase

- Un $89,2 \%$ para consultar dudas sobre las tareas propuestas

- Un 74,6\% para consultar fechas importantes (exámenes, entrega de trabajos, etc.)

- Un 6\% lo utiliza para otras cuestiones, entre las que señala quedar para realizar algún trabajo, cambio de horarios o aulas. 
Los datos recogidos en este momento previo, nos recuerdan, como ya explicaba Sanz (2014) el lugar relevante que esta aplicación ha ocupado en nuestras vidas y en la vida de los jóvenes, y confirma que los estudiantes mantienen sus propios grupos dentro de las distintas redes sociales y mensajerías instantáneas para estar al día de cuestiones académicas y ayudarse (Mosquera, 2016; Rubio y Perlado, 2015; Sanz, 2014).

Como explican Merelo y Tricas (2012, p. 219):

Muchísimos, si no la mayoría, de las clases del país tienen su WhatsApp "de clase" en el que intercambian cotilleos, los alumnos que faltan se enteran de qué se ha dicho y, en general, se hacen todas las tareas que se esperan de una sala de chat de grupo.

En una segunda parte, y una vez presentada la aplicación como herramienta de apoyo a la tutoría, los resultados obtenidos son los siguientes:

\section{(iii) ¿Qué uso dan a la aplicación como apoyo a la tutoría?}

El uso dado a la herramienta, según el registro de mensajes, ha sido para resolver dudas sobre la elaboración de tareas (individuales o grupales), fechas de entrega, formato, citas y referencias o bibliografía y, en menor medida, dudas sobre cuestiones teóricas vistas en el aula o el formato del examen final.

Estos resultados no hacen más que corroborar las afirmaciones realizadas por autores como Alonso et al. (2018), Amor y Dios (2017) o Sanz (2009) sobre el uso que los estudiantes dan a la tutoría académica, quienes señalan que se dedica principalmente a la resolución de dudas, revisión de exámenes, trabajos u otros aspectos relacionados con la materia que se imparte. A pesar de que, como explican Alonso et al. (2018, p. 65):

La tutoría no debe entenderse como una función complementaria a la docencia, sino como un todo desde el que se abordarían varios ámbitos-contenidos (burocráticos, académicos, personales y profesionales) para dar respuesta a las necesidades que van surgiendo a lo largo de la trayectoria académica del estudiante.

Los estudiantes han utilizado frecuentemente el grupo para sus consultas. Como ya indicaba Mosquera (2016, p.3): "Si usamos los mismos canales de comunicación que ellos emplean en su vida diaria, cabe esperar que la comunicación sea más frecuente, continua y fluida".

Si bien el uso dado ha sido correcto (no hubo lenguaje o mensajes inadecuados) algunos estudiantes pasaron de realizar consultas en el grupo a realizarlas de forma privada. Aunque este hecho sucedió con una minoría de estudiantes y las consultas, en algunos casos, estaban relacionadas con cuestiones personales (no asistencia a clases, cambio de horario, etc.).

Esta tendencia confirma, también en el formato virtual, la preferencia del alumnado hacia la tutoría personal sobre la grupal y virtual (Martínez, Pérez y Martínez, 2014)

No obstante, este resultado también fue observado por Suárez (2017) quien expresa que esta cuestión (en opinión del docente) perjudica la interacción del grupo, puesto que la duda planteada por un estudiante no se resolvía en el grupo, evitando que se compartiese la respuesta $\mathrm{y}$, por tanto, en alguna ocasión las cuestiones que se planteaban eran repetidas, tanto de modo privado o personal como en el grupo.

En relación con el tiempo, señalar que los estudiantes han realizado un uso correcto. No se recibieron consultas en horario inoportuno, pero sí durante el fin de semana (en fechas previas a la entrega de trabajos o tareas). 


\section{(iv) ¿Es satisfactoria la experiencia para los participantes?}

El docente se muestra complacido con el uso de la aplicación, pues los estudiantes se animaron a realizar más consultas (de las que suelen hacer a través de las otras vías de tutoría). Además, este incremento se vio reflejado en una mejora de la calidad de los trabajos que los estudiantes entregaban (y por tanto en la calificación final de la materia), es decir, en sus resultados de aprendizaje.

Estos datos confirman lo expuesto por Pozo e Iglesias (2013) la implementación de Internet y el incremento de plataformas online, favorece la comunicación entre el estudiante y el profesorado pues no es necesaria la presencia física, establecer horas determinadas para quedar, etc.

En la misma línea, López y Prendes (2017) manifiestan, sobre la tutoría electrónica, que permite superar algunos inconvenientes de la tutoría presencial al flexibilizar las condiciones de espacio y tiempo -tanto para estudiantes como para el profesorado- algo que también puede aplicarse en este caso.

Aunque no debemos olvidar que si queremos que este tipo de recursos funcionen de forma efectiva como vía de comunicación entre profesorado y alumnado, se precisa que el docente conteste dudas individuales o colectivas con cierta inmediatez, ya que si se demora demasiado los estudiantes dejarán de usarlo (Mosquera, 2016; Suárez, 2017).

De hecho, en la fase de grupo control se corrobora que el grupo que contaba con la aplicación efectuó más consultas (a través de este medio) que el grupo que no contaba con el apoyo de WA y debía asistir a tutorías presenciales o utilizar la tutoría virtual.

En todas las fases del proyecto, la mayoría de los estudiantes (entre el 98\% y el 100\%, según las fases) mostraron satisfacción con la inclusión de la aplicación, pues permitía resolver sus dudas de forma rápida y eso les permitía seguir con el trabajo o estudio. No obstante, una minoría (2\%) expresa que estar pendiente de los mensajes supone perder tiempo que podrían emplear para otras cuestiones.

Estos datos coinciden con los recogidos en el estudio de Gómez, Roses y Farias (2012) donde más de la mitad de los estudiantes valoraron positivamente la posibilidad de crear un grupo de las asignaturas en alguna red social.

\section{(v) Las ventajas y desventajas}

Los cuestionarios iniciales resueltos por los estudiantes presentaban como ventaja principal el favorecer la solución de dudas (100\% de los participantes), seguida de mejorar en el desarrollo y presentación de las tareas y trabajos (96\%). En cuanto a las desventajas, se señala el tiempo que implica atender a "tantos grupos de WA" y el temor a que se presenten consultas o mensajes que no sean interesantes.

En el cuestionario final se confirman estas ventajas, añadiendo, por parte del docente, la mejora de la participación, observando la posibilidad de atender mejor a la diversidad del alumnado que, normalmente, no realiza tantas consultas por las vías ordinarias de tutoría. En el caso de las desventajas, el docente siente que se puede uno sentir un poco "agobiado" si se reciben varias consultas en un periodo de tiempo corto, teniendo la sensación de que debe estar "disponible" en cualquier momento.

Las ventajas y desventajas indicadas por estudiantes y docentes, tanto en el cuestionario inicial como en el cuestionario final, vienen a agruparse en las categorías que se recogen en la tabla 1. 
Tabla 1.

Ventajas y desventajas observadas en el uso de WA

\begin{tabular}{|c|c|}
\hline VENTAJAS & DESVENTAJAS \\
\hline $\begin{array}{l}\text { - Favorece la interacción y comunicación entre } \\
\text { docente y estudiantes } \\
\text { - Favorece la participación. Invita a que se } \\
\text { consulten más dudas } \\
\text { - Genera unión entre los estudiantes, } \\
\text { sensación de grupo-clase } \\
\text { - Favorece la atención a todo el alumnado, por } \\
\text { tanto la atención a la diversidad } \\
\text { - Resolver las dudas sobre la materia favorece } \\
\text { mejores resultados en el proceso de } \\
\text { enseñanza-aprendizaje. } \\
\text { - Los estudiantes consideran que sus dudas se } \\
\text { resuelven "prácticamente en el momento" lo } \\
\text { que ayuda en la elaboración de los trabajos y } \\
\text { tareas. }\end{array}$ & $\begin{array}{l}\text { - El docente debe utilizar su número privado } \\
\text { para el desarrollo de la experiencia. Este hecho } \\
\text { genera cierta desconfianza por si pudiera darse } \\
\text { otra utilidad a dicha información. } \\
\text { - El tiempo dedicado por el docente para dar } \\
\text { respuesta a las consultas } \\
\text { - La realización de consultas por parte de } \\
\text { algunos estudiantes de modo privado, en vez } \\
\text { de realizarlas a través del grupo } \\
\text { - A pesar de que, en este caso concreto, el uso } \\
\text { por parte de los estudiantes fue correcto, se } \\
\text { puede establecer la creencia entre los } \\
\text { estudiantes de que el profesor debe estar } \\
\text { disponible en cualquier momento del día o de la } \\
\text { semana. }\end{array}$ \\
\hline
\end{tabular}

Estas ventajas y desventajas, en líneas generales, coinciden con las señaladas en otros estudios que también han puesto su objetivo en la aplicación WhatsApp para fines educativos (Bottentuir y Patriota, 2016; Bouhnik y Deshen, 2014; Sanz, 2014; o Suárez, 2018).

Debemos señalar también que los datos observados tras la firma de un compromiso de uso por parte del alumnado (en la última fase del proyecto) mostraron una solución a que el alumnado realice un aprovechamiento óptimo: no se enviaron mensajes inadecuados y las horas de las consultas fueron pertinentes.

\section{Conclusiones}

Tras los resultados presentados podemos concluir que WA resulta ser, no solo como presentan Bouhnik y Deshen (2014) una herramienta con potencial educativo y académico, también una herramienta adecuada de apoyo a la tutoría académica.

Dado que, tanto las docentes como los estudiantes muestran un nivel de satisfacción elevado con el uso de esta aplicación como herramienta de apoyo la tutoría académica, podemos recomendar su uso a otros docentes.

Entre las ventajas más valoradas por los estudiantes está el hecho de que permite obtener respuesta a sus dudas de forma rápida y global.

En relación con las desventajas observadas y la solución propuesta en este proyecto para salvar la reticencia mostrada por los docentes en cuanto al uso de número personal y la recepción de mensajes no apropiados o en horario no adecuado, valoramos positivamente el hecho de que los estudiantes firmasen un compromiso para poder participar. Esta cuestión favoreció un buen uso, siendo las intervenciones adecuadas tanto en forma como en tiempo. Por tanto, se aconseja establecer unas normas de uso antes de tomar esta iniciativa. 
No obstante, y a pesar de los buenos resultados observados, la experiencia también ha contado con limitaciones, algunas de las cuales coinciden con las que otros autores, como López y Prendes (2017) o Pérez Cusó (2013) observan para los sistemas de tutoría electrónica o virtual:

- La necesidad de contar en el acceso a las tecnologías (y Smartphone, en este caso) de estudiantes con menos recursos

- Las dificultades para establecer una relación de confianza mutua y participar en un grupo en el que se incluya al docente.

- La necesidad de establecer un compromiso en el mantenimiento de la comunicación por ambas partes. El docente para responder con relativa inmediatez y el alumnado para no realizar consultas inapropiadas en modo y tiempo.

Otras son más específicas de este estudio:

- Los cuestionarios, diseñados para la propia experiencia, debían de pasar por procesos que de validación y fiabilidad con el fin de conceder mayor consistencia a la metodología utilizada.

- Debemos considerar que el uso dado ha sido en grupos no muy numerosos (no más de 75 alumnos/as/grupo), por tanto, no sabemos cómo resultaría tener que responder dudas en aquellos grupos con un número de estudiantes más elevado o para un mismo docente con varios grupos numerosos.

Por ello, consideramos la necesidad de seguir trabajando en el campo de la tutoría y la inclusión de herramientas que la fortalezcan, pues no solo es un instrumento fundamental en el proceso de enseñanza-aprendizaje y apoyo para los estudiantes, también:

Las acciones tutoriales mejoran la imagen pública y proyección externa de la universidad, así como promueven una valoración positiva del alumnado de la propia institución [...] con lo cual su utilidad no es solamente individual sino también organizativa e institucional (López y Prendes, 2017, p. 261)

\section{Referencias}

Alonso, S., Rodríguez, A.M. y Cáceres, M.P. (2018). Análisis de la acción tutorial y su incidencia en el desarrollo integral del alumnado. El caso de la Universidad de Castilla-La Mancha, España. Formación universitaria, 11 (3), 63-72, doi: 10.4067/So71850062018000300063

Álvarez, M. y Álvarez, J. (2015). La tutoría universitaria: del modelo actual al modelo integral. Revista electrónica interuniversitaria de formación del profesorado, 18 (2), 125-142.

Amor, M.I. y Dios, I. (2017). La tutoría universitaria: un espacio para la orientación personal, académica y profesional en la formación inicial del profesorado. REOP, 28 (1), 119-130.

Barhoumi, C. (2015). The effectiveness of WhatsApp mobile learning activities guided by activity theory on students' knowledge management. Contemporary Educational Technology, 6 (3), 221-238.

Bottentuit, J.B. y Patriota, O.C. (noviembre, 2016). Possibilidades para uso do WhatsApp na educaçao: Analise de casos e estrategias pedagógicas. Comunicación presentada en el I Simpósio Nacional de Tecnologías Digitais na Educaçao, celebrado en Sâo Luis (Brasil), en noviembre de 2016. Accesible siguiendo el enlace: 
https://lccp.ufra.edu.br/images/doc/Anais_Tecnologias_Digitais_na_Educa\%C3\%A7\%C 3\%A30.pdf

Bouhnik, D. y Deshen, M. (2014). WhatsApp goes to school: Mobile instant messaging between teacher and student. Journal of Information Technology Education: Research, (13), 217-231, doi: 10.28945/2051

Da Re, L. y Clerici, R. (2017). Abandono, rendimiento académico y tutoría: una investigación de la Universidad de Padua. Educatio Siglo XXI, 35 (2), 139-160, doi: 10.6018/j/298551

Ditrendia (2018). Informe Mobile en España y en el mundo 2019. Documento on-line, accesible siguiendo el enlace: https://ditrendia.es/informe-mobile-2018/

Echeverría, B. y Martínez, P. (2015). Luces entre sombras de la orientación. Revista electrónica interuniversitaria de formación del profesorado, 18 (2), 1-13.

García, B. (2010). La tutoría en la universidad de Santiago de Compostela: percepción y valoración de alumnado y profesorado. Tesis doctoral. Santiago de Compostela: Universidad de Santiago de Compostela.

Gende, I. M. (2016). Creación de comunidad y comunicación mediante el uso de Whatsapp en la elaboración online de Trabajos Fin de Máster de Formación de Profesorado. DIM: Didáctica, Innovación y Multimedia, (33), 1-8

Gómez, M., Roses, S. y Farias, P. (2012). El uso académico de las redes sociales en universitarios. Comunicar, doi: 10.3916/C38-2011-03-04

López, P. y Prendes, M.P. (2017). Estudio longitudinal sobre tutoría académica flexible en la universidad. Profesorado, 21 (4), 259-278.

Martínez, P., Pérez, J. y Martínez, M. (2014). Una (re)visión de la tutoría universitaria: la percepción de estudiantes y tutores de estudios de Grado. Revista de Docencia Universitaria, 12 (1), 261-305, doi: 10.4995/redu.2014.6425

Martínez, M. E. y Raposo, M. (2006). Las TIC en manos de los estudiantes universitarios. Revista Latinoamericana de Tecnología Educativa, 5(2), 165-176

Medialdea, A.L. (2014) Motivos y factores explicativos del abandono en el ámbito de las ciencias sociales. Tesis doctoral. Sevilla: Universidad Pablo de Olvaide. Accesible en: https://rio.upo.es/xmlui/handle/10433/888

Merelo, J. J. y Tricas, F. (2012). La irresistible ascensión de WhatsApp. ReVisión, 6 (2), 3-4.

Mosquera, I. (2016). Creación de comunidad y comunicación mediante el uso del WhatsApp en la elaboración on-line de Trabajos Fin de Máster de Formación del Profesorado. Didáctica, innovación y multimedia (DIM), (33), 1-8.

Pérez Cusó, F.J. (2013). Tutoría universitaria: ¿un elemento de calidad? Un estudio en la Facultad de Educación de la Universidad de Murcia, Murcia. Accesible en: https://digitum.um.es/digitum/bitstream/10201/36176/1/Tesis\%20Javier\%20P\% c3\%a9rez\%20Cus\%c3\%b3.pdf

Pozo, J.S. e Iglesias, C. (2013). Evaluación del empleo de las TIC por parte del alumnado de la Universidad de Vigo. Revista de Formación e Innovación Educativa Universitaria, 6 (2), 80-87.

Rodríguez, M. C., Valerio, G., Cárdenas, C. A., y Herrera, D. J. (2016). Percepción y realidad del uso de WhatsApp en estudiantes universitarios de ciencias de la salud. FEM: Revista de la Fundación Educación Médica, 19 (3), 118-124. 
Rubio, J. y Perlado, M. (2015). El fenómeno WhatsApp en el contexto de la comunicación personal: una aproximación a través de los jóvenes universitarios. Icono 14, (13), 7394, doi: $10.7195 /$ ri14.v13i2.818

Sanz, R. (2009). Tutoría y atención personal al estudiante en la Universidad. Madrid, España: Síntesis

Sanz, J.J. (2014). WhatsApp: potencialidad educativa versus dependencia y adicción. Revista didáctica, innovación y multimedia (DIM), (30), 1-8.

Sonlleva, M., Torrego, A. y Martínez, S. (2017). "Es una locura vivir sin Facebook o WhatsApp": la huella tecnológica en el docente en formación. Edmetic, 6 (2), 255276, doi: 10.21071/edmetic.v6i2.6935

Suárez, B. (2017). WhatsApp como herramienta de apoyo a la tutoría. Revista de Docencia Universitaria, 15 (2), 193-210.

Suárez, B. (2018). WhatsApp: su uso educativo, ventajas y desventajas. Revista de Investigación en Educación, 16 (2), 2018, 121-135. 


\section{Anexo I}

\section{CUESTIONARIO PRE-USO WHATSAPP PARA ALUMNADO}

EDAD:

GÉNERO:

ESTUDIOS QUE CURSAS (nombre del grado o postgrado)

CURSO:

N DE ASIGNATURAS DE LAS QUE ESTÁS MATRICULADO:

1. ¿Utilizas tu móvil para estar conectado con otras personas? Sí NO

2. ¿Cuántas horas al día consideras que estás conectado?

3. ¿Utilizas algún sistema de mensajería en tu móvil? Sí NO

En caso afirmativo, indica cuáles:

4. ¿Utilizas el teléfono móvil para intercambiar información sobre el curso, las asignaturas, los contenidos, etc.? Sí NO

En caso afirmativo, señala con una $X$ con qué personas intercambias la información:

- Amigos

- Compañeros

- Docentes

- Tutores

- Otros (indicar quién):

5. ¿Qué aplicaciones utilizas en mayor medida para ello? (señala con una X)

- WhatsApp

- Line

- Instagram

- Twitter

- Facebook/messenger

- Otros (señalar):

6. Indica para qué haces uso de tu móvil en relación con las materias que cursas: (señala con una X)

- Informarte de los contenidos impartidos en clase

- Consultar dudas sobre lo explicado en clase

- Consultar dudas sobre las tareas propuestas

- Consultar fechas importantes (exámenes, entrega de trabajos, etc.)

- Otros (señalar):

7. ¿En cuántas asignaturas tienes grupos de WhatsApp con tus compañeros de clase?

Indica el número: 
8. ¿Está incluido el profesor en alguno de ellos? Sí NO

En caso afirmativo, indica el número de grupos:

9. ¿Consideras útil el uso de aplicaciones como el WhatsApp en tu proceso de aprendizaje? Sí NO

10. ¿Consideras que el uso de aplicaciones como el WhatsApp en tu proceso de aprendizaje tiene desventajas? Sí NO

Señala las ventajas y desventajas que encuentras:

\begin{tabular}{|l|l|}
\hline VENTAJAS & DESVENTAJAS \\
\hline & \\
& \\
\hline
\end{tabular}

\section{Anexo II}

\section{CUESTIONARIO FINAL USO DE WHATSAPP COMO APOYO A LA TUTORÍA}

1. Expresa tu opinión sobre el empleo del grupo de WhatsApp en el desarrollo de la asignatura

2. ¿Consideras útil el uso de aplicaciones como el WhatsApp en tu proceso de aprendizaje? Sí NO

3. Señala las ventajas y desventajas que observas:

\begin{tabular}{|l|l|}
\hline VENTAJAS & DESVENTAJAS \\
\hline & \\
1 & 1 \\
& 1 \\
\hline
\end{tabular}

\section{Anexo III}

\section{DOCUMENTO PERMISO DATOS Y COMPROMISO DE CONFIDENCIALIDAD}

Yo: (nombre y apellidos)

Acepto compartir mi número de teléfono en el grupo de WhatsApp creado para la asignatura.

Me comprometo a no utilizar la información (números de teléfono y contenido compartido) en otros contextos que no sea el establecido en la creación de dicho grupo:

1. El grupo se crea como complemento a la tutoría académica 
2. Su utilización será con fines académicos (compartir documentos propios de la asignatura, consultas en referencia a trabajos o contenidos, seminarios, jornadas, fechas relevantes, evaluación...) u otras cuestiones que surjan a lo largo del proceso enseñanza-aprendizaje

3. La participación en el grupo es voluntaria: pertenecer a dicho grupo no implica tener que participar

4. Esta participación se hará en horario lectivo (de lunes a viernes, mañanas y/o tardes)

5. Los mensajes compartidos serán respetuosos, adecuados en su redacción y contenido

6. Cualquier miembro del grupo puede participar en la solución de dudas para las que conozca la respuesta correcta, siendo labor del docente comprobar que dicha respuesta es veraz

7. El hecho de utilizar el grupo no impide que las dudas puedan ser aclaradas en el aula

Y para que así conste:

Firmado:

Los datos de carácter personal que se recaben a través de este grupo serán sometidos a tratamiento en los términos de la Ley 39/2015, de 1 de octubre, de Procedimiento Administrativo Común de las Administraciones Públicas, que sirve de base jurídica para el tratamiento. Se conservarán durante el tiempo que necesario para cumplir con la finalidad para la que se recabaron y para determinar las posibles responsabilidades que se pudieran derivar de dicha finalidad y del tratamiento de los datos. Será de aplicación lo dispuesto en la normativa de archivos y documentación. Si desea solicitar más información sobre cualquier aspecto relativo al tratamiento de sus datos personales podrá dirigirse a la dirección mariabelen.suarez@um.es, irinasherezade.castillo@um.es, almedialdez@unex.es 